\title{
Health Outcomes and Cost Benefit of Home e-Health in Helping to Manage Heart Failure
}

\author{
Yuan $\mathrm{Yao}^{1}$ and $\mathrm{Ying} \mathrm{Su}^{2}$ \\ ${ }^{1}$ Military Medical Sciences and PLA General Hospital, China \\ ${ }^{2}$ Institute of Scientific and Technical Information of China, China, \\ 100038, Beijing, China \\ yaoyuan301@sina.cn, suy.rspclistic.ac.cn
}

\begin{abstract}
This study presents a systematic review of the nature and magnitude of outcomes associated with e-Health of cardiac diseases. Methods: A comprehensive literature search was conducted on Medline and the Cochrane Library to identify relevant articles published between 2000 and 2014 in which the outcome measure used was one or more of health outcome, proxy health outcome, patient compliance or cost. Studies identified were subjected to narrative review. The magnitude and significance of the Home e-Health effects on patients' conditions (e.g., early detection of symptoms, decrease in blood pressure, adequate medication, reduced mortality) still remain inconclusive for cardiac diseases. However, the results of this study suggest that regardless of their nationality, socioeconomic status, or age, patients comply with Home eHealth programs and the use of technologies. Importantly, the Home e-Health effects on clinical effectiveness outcomes (e.g., decrease in the emergency visits, hospital admissions, average hospital length of stay) are more consistent in Heart Failure. Home e-Health of cardiac diseases seems to be a promising patient management approach that produces accurate and reliable data, empowers patients, influences their attitudes and behaviors, and potentially improves their medical conditions. Future studies need to build evidence related to its clinical effects, cost effectiveness, impacts on services utilization, and acceptance by health care providers.
\end{abstract}

Keywords: Chronic heart failure, Blood pressure control, Lung transplantation, Monitoring system, Pulmonary function, Diabetes control, Self-measurement, Controlled trial, Hypertensive patients, African Americans.

\section{Introduction}

It is over a decade since e-Health started to be explored as a tool in the reduction of exposure to risk factors for disease, and in the management of illness. It has taken various forms as technology has evolved, but with the exception of the remote interpretation of images, has failed to become part of mainstream medicine in, for instance, the UK. In previous research, most of intelligent works were centralized at healthcare center and the function of telemedicine terminal device was mainly 
focused on the acquisition and transmission of physiological data. Heart Failure has features which appear to make it particularly suitable for management involving eHealth: measurement in healthcare settings may be spuriously high ("white coat cardiac diseases"); people with mild to moderate cardiac diseases feel well when off medication but may feel unwell on treatment so may not use it conscientiously; the information to be conveyed between the individual and the healthcare professional is simple; the individual's use of the equipment is unlikely to be comprised by their being acutely anxious or fearful because of the condition. We created a generic workflow model based on interviews and observations at three CR clinics.

\section{Methods}

A comprehensive literature search was conducted on Medline and the Cochrane Library to identify relevant articles published between 2003 and 2013. The keywords that were used include telemonitoring, telecare, telemedicine, telematics, telehealth, and telehomecare.. All abstracts were read to identify how many studies were reported in the literature. The inclusion criteria required that the studies: (1) have an experimental design involving direct data collection from patients with any of the four considered chronic diseases, (2) be published in the English language and appear in peer-reviewed journals, and (3) document telemonitoring effects. The abstracts and papers were reviewed by two independent readers.

Table 1. Overview of Research Designs

\begin{tabular}{ll}
\hline Sensor type & Cardiac Diseases \\
\hline Total number of studies & 16 \\
Type of design & 7 \\
Adults with congestive heart failure & 15 \\
Size of experimental group & Minimum:10; Maximum 230 \\
Study duration & Minimum:1 month; Maximum 36 months \\
Main types of data being transferred & Basic vital signs; Symptoms \\
Frequency of data transmission & More than once a day 4, Once a day 8 \\
\hline
\end{tabular}

\section{Results}

\subsection{Literature Search}

Forty three abstracts were found, and led to the identification of 14 relevant RCTs. The 29 abstracts were rejected because they did not report information on health outcome, proxy health outcomes, treatment process, patient compliance and/or cost with particular respect to management of cardiac diseases. Some were excluded as they were trialing mobile phone-based interventions with no e-Health element. 


\subsection{Health Outcome}

With the exception of pulmonary diseases that represent a variety of medical conditions, telemonitoring of cardiac diseases was specific to each of these illnesses, and involved adult patients suffering from these medical conditions. Only in few cases, subgroups of the general population (e.g., children, veterans, pregnant women) were considered.

\subsection{Proxy Health Outcome}

Four RCTs evaluated the efficacy of e-Health in controlling cardiac diseases, with somewhat conflicting results.

Kirtava, Z., et al 2013[1] randomised 54 patients with main medical results, Quality of Service (QoS), Quality of Experience (QoE), cost-efficiency and remaining challenges - is presented, as well as the outline of its continuation - MTM-2 project, which aims improvement of decision making for emergency cardiac patients and usage of mHealth applications for integrated care provision in remote regions of Georgia. This result was confirmed by two other RCTs. Goroso, D. G et al 2013 [2] provide a mobile tool to prevent early cardiac arrhythmia by means of monitoring online and assessing heart rate variability. Preliminary results proved effective in Sao Paulo for study of heart rate variability. This new service uses mobile phone to record, evaluate and transmit information, which currently is in clinical validation. Foche-Perez et al 2012 [3] twelve patients have been auscultated by all the physicians using the tele-stethoscopy system, versus a local auscultation using traditional stethoscope. The system must allow listen the cardiacpatientspatients (systolic and diastolic murmurs, gallop sound, arrhythmias) and respiratory (rhonchi, rales and crepitations, wheeze, diminished and bronchial breath sounds, pleural friction rub) sounds[3].

An architectural framework of a system utilising mobile technologies to enable continuous, wireless, electrocardiogram (ECG) monitoring of cardiac patients. The proposed system has the potential to improve patients' quality of life by allowing them to move around freely while undergoing continuous heart monitoring and to reduce healthcare costs associated with prolonged hospitalisation, treatment and monitoring[4]. At follow up, $36 \%$ of those allocated to 'e-Health' had pressures below $140 / 90$ as did $31 \%$ of those receiving usual care. Only those in the third arm -'eHealth' as above plus web delivered pharmacist support - did significantly better than those receiving usual care (56\% controlled) [5].

\subsection{Process of Care}

Telemonitoring projects of cardiac diseases had the largest samples of patients, which exceeded by far the size of the experimental and the largest average study durations (Table 1).

Fanucci et al 2013 [6] evaluated a complete and integrated Information and Communication Technology system (CHF patients to daily collect vital signs at home 
and automatically send them to the Hospital Information System, allowing the physicians to monitor their patients at distance and take timely actions in case of necessity.) against usual care in confirming suspected cardiac diseases in 74 adults. All 74 underwent 24 hour ambulatory monitoring; e-Health appeared superior in detecting true cases $(64 \%)$ to usual care $(26 \%)$. E-Health was also more rapid in confirming or refuting the suspicion of cardiac diseases.

Nowadays, chronic heart failure (CHF) affects an ever-growing segment of population, and it is among the major causes of hospitalization for elderly citizens[6]. Dennis et al 2013 [7] addressed Telephone coaching for people with chronic conditions can improve health behaviour, self-efficacy and health status: telemedical home blood pressure measurement ( 5 days, duplicate measurement, four times daily), clinic blood pressure or ambulatory blood pressure monitoring. They confirmed the existence of "white coat cardiac diseases", but showed an increasingly good correlation between telemedical and ambulatory monitoring over the five day periods, with the afternoon readings having the best correlation with the ambulatory value. Cowie et al 2013 [8] performed an equivalence study to e-Health innovation in detecting uncontrolled cardiac diseases over one year in 62 out-patients of a cardiac diseases clinic. Each patient had e-Health and face-to-face consultations on the same days, the order of the two encounters being randomised. The e-Health system was found to be equivalent to in-person consultation in detecting uncontrolled cardiac diseases, in the physicians' use of additional tests, and in the associated therapeutic decisions. ESC has invested in innovation in education and learning by delivering rich content from its meetings online, by delivering a variety of specialty courses on webinars, and last but not least by delivering clinical guidelines to the personal digital assistants of specialists.. Munoz et al 2011 [9] explored a case series of pediatric patients teleassisted from the Cardiac Intensive Care Unit (CICU) at Children's Hospital of Pittsburgh of University of Pittsburgh Medical Center, Pittsburgh, PA, to the CICU at Hospital Valle del Lili, Cali, Valle, Colombia, between March and December 2010. Seventy-one recommendations were given regarding 53 patients. Median age and weight were 10 months and $7.1 \mathrm{~kg}$, respectively. Ventricular septal defect, transposition of the great vessels, and single ventricle accounted for most cases. The most frequent recommendations were related to surgical conduct, management of arrhythmias, and performance of cardiac catheterization studies. No technical difficulties were experienced during the monitoring of the patients. Satisfaction rates were equally high for technical and medical aspects of telemedicine service.

Duck Hee et al 2012 [10] showed that the fall in blood pressure in their e-Health group was (ECG) signal preprocessing including filtering, power noise canceling, and level shifting adherence of $17.7 \%$.

\subsection{Cost of Care}

Kifle, M., et al. [11] examines the role of Tele-Medicine in the healthcare system and analyzes the costs and benefits of introducing Tele-Cardiology services in Ethiopia (a Sub- Saharan African country). This is a cost comparison study for the treatment of 
cardiac patients traveling abroad versus patients treated via Tele-Cardiology. Our findings show that Tele-Cardiology is clinically more feasible and more cost effective compared to patients traveling abroad for treatment. Those monitoring themselves made on average 1.2 fewer cardiac diseases related consultations in the year which represented a cost saving despite the expense of the equipment. Martin et al 2013 [8] need to be involved, and to collaborate with engineers and other key stakeholders at each stage of the process: the technology design and integration, the assessment of the value of e-health innovation (in terms of the impact on patients, the healthcare system, and the costs), the education and support of healthcare professionals and patients, the development of guidelines, and the standardization of systems and models.

\section{Discussions}

The introduction of e-Health is a change to the structure of healthcare, so it is appropriate to consider its effect on the processes and outcomes of care. This survey of papers reporting RCTs shows that it is feasible, at least experimentally, to introduce e-Health into the process of identifying and working with individuals with cardiac diseases to reduce their subsequent risk of stroke, ischaemic heart disease and sudden death. It also confirms that this has not been introduced into everyday clinical practice in countries which take an 'evidence based' approach to healthcare, though the literature has developed [12-14]from that available to the guideline development groups producing the CSIRO (Commonwealth Scientific and Industrial Research Organisation) and the Queensland Government have jointly established the Australian e-Health Research Centre (AEHRC) Guideline 97 (Risk estimation and prevention of cardiovascular disease February 2011). The former guideline says "Routine use of automated ambulatory blood pressure monitoring or home monitoring devices in primary care is not currently recommended because their value has not be adequately established; appropriate use in primary care remains an issue for further research" and the latter does not mention it at all.

There is no definitive evidence that e-Health improves the ultimate health outcomes of the management of cardiac diseases. Our synthesis of the literature indicates that e-Health is likely to be beneficial in controlling cardiac diseases, and thus has the potential to improve health outcomes. Little is known about how this effect is mediated - there is one suggestion that it is through improved compliance. EHealth can also reduce the requirement of hospital care and the number of mortalities. Modern e-health systems can control successfully such diseases as cardiac, diabetic or pulmonary diseases.

The major weakness of this paper is that it is narrative review not a systematic review or a meta-analysis. This is compounded by the fact that the RCTs available are generally small and/or have short follow-up times, so should not be over-interpreted. A more quantitative approach would add value, but, given that there are some relevant trials that have finished recruiting participants but not yet reported (The European Commission has undertaken to support member states in deploying e-health solutions for chronic disease management and in setting quantitative targets such as reduction 
in hospitalization for heart failure, reduction in healthcare resources by patients with diabetes, and improvement in quality and length of life.), there will be a more optimal opportunity for formal meta-analysis in the near future. We also note that Fayn et al are about to start another trial building an operational e-health infrastructure including pervasive services and remote distributed applications servers in the cardiology domain[15].

\section{Conclusions}

So far, despite the recent history of home e-Health, a significant body of knowledge has been developed and made available to policymakers and clinicians. Based on the results of this review, home e-Health of cardiac diseases seems to be a promising patient management approach that produces accurate and reliable data, empowers patients, influences their attitudes and behaviors, and potentially improves their medical conditions. Nevertheless, some studies have shown that there is an overall reduction in both systolic and diastolic values, and the presumed mechanism is better patient engagement in disease control and compliance. For insurance companies and governments to consider future endorsement of this patient management approach, and subsequent reimbursement for the services provided, it is important to demonstrate its feasibility at the population level. More rigorous research on home eHealth would build stronger evidence that lead to changes in the practice and management of these cardiac illnesses, to acceptance of this patient management approach by payers and providers, and to its future integration in the overall process of care.

Acknowledgements. The researchers acknowledge the support of the National Soft Science Planning (2011GXQ4K029), and National Population and Reproductive Health Science Data Center of National Scientific Data Sharing Platform for Population and Health funded by Ministry of Science and Technology, China; the research volunteers and the respondees.

\section{References}

1. Kirtava, Z., Gegenava, T., Gegenava, M.: mHealth for cardiac patients telemonitoring and integrated care. In: 2013 IEEE 15th International Conference on E-Health Networking, Applications and Services (Healthcom 2013), pp. 5-21 (2013)

2. Goroso, D.G., da Silva, R.R., Battistella, L.R., Odstrcil, M., Paolini, M.: Monitoring heart rate variability on-line used mobile telephone $3 \mathrm{G}$ e-health service oriented. Journal of Physics: Conference Series 477, 12036(8 pp.) (2013)

3. Foche-Perez, I., Ramirez-Payba, R., Hirigoyen-Emparanza, G., Balducci-Gonzalez, F., Simo-Reigadas, F.-J., Seoane-Pascual, J., et al.: An open real-time tele-stethoscopy system. Biomedical Engineering Online 11 (August 23, 2012)

4. Sneha, S., Varshney, U.: A wireless ECG monitoring system for pervasive healthcare. International Journal of Electronic Healthcare 3, 32-50 (2007) 
5. Pare, G., Jaana, M., Sicotte, C.: Systematic review of home telemonitoring for chronic diseases: The evidence base. Journal of the American Medical Informatics Association 14, 269-277 (2007)

6. Fanucci, L., Saponara, S., Bacchillone, T., Donati, M., Barba, P., Sanchez-Tato, I., et al.: Sensing Devices and Sensor Signal Processing for Remote Monitoring of Vital Signs in CHF Patients. IEEE Transactions on Instrumentation and Measurement 62, 553-569 (2013)

7. Dennis, S.M., Harris, M., Lloyd, J., Powell, G., Faruqi, N., Zwar, N.: Do people with existing chronic conditions benefit from telephone coaching? A rapid review. Australian Health Review 37, 381-388 (2013)

8. Cowie, M.R., Chronaki, C.E., Vardas, P.: E-Health innovation: time for engagement with the cardiology community. European Heart Journal 34, 1864-1868 (2013)

9. Munoz, R.A., Burbano, N.H., Motoa, M.V., Santiago, G., Klevemann, M., Casilli, J.: Telemedicine in Pediatric Cardiac Critical Care. Telemedicine and E-Health 18, 132-136 (2012)

10. Duck Hee, L., Rabbi, A.F., Root, N., Fazel-Rezai, R., Jaesoon, C., de Leon, P., et al.: A heart monitoring system for a mobile device. International Journal of Handheld Computing Research 3, 22-39 (2012)

11. Kifle, M., Mbarika, V.W.A., Datta, P.: Interplay of cost and adoption of tele-medicine in Sub-Saharan Africa: The case of tele-cardiology in Ethiopia. Information Systems Frontiers 8, 211-223 (2006)

12. Hickey, K.T., Johnson, M.P., Biviano, A., Aboelela, S., Thomas, T., Bakken, S., et al.: Cardiac e-Learning: Development of a Web-Based Implantable Cardioverter Defibrillator Educational System. Telemedicine and E-Health 17, 196-200 (2011)

13. Hansen, D.P., Gurney, P., Morgan, G., Barraclough, B.: The Australian e-Health Research Centre: enabling the health care information and communication technology revolution. Medical Journal of Australia 194, S5-S7 (2011)

14. Karunanithi, M., Varnfield, M., Ding, H., Garcia, E., Whittaker, F., Sarela, A., et al.: Care Assessment Platform: An ICT-Enabled Home Care Model for Secondary Prevention of Cardiovascular Diseases. In: 2010 Annual International Conference of the IEEE Engineering in Medicine and Biology Society, pp. 5266-5266 (2010)

15. Fayn, J., Ghedira, C., Telisson, D., Atoui, H., Placide, J., Simon-Chautemps, L., et al.: Towards new integrated information and communication infrastructures in e-health. Examples from cardiology, Computers in Cardiology 2003 (IEEE Cat. No.03CH37504), $113-116(2003)$ 